for

\title{
Using Visible Laser-Based Raman Spectroscopy to Identify the Surface Polarity of Silicon Carbide
}

\author{
Submitted by \\ Yi-Chuan Tseng, ${ }^{a}$ Yu-Chia Cheng, ${ }^{a}$ Yang-Chun Lee, ${ }^{a}$ Dai-Liang Ma, ${ }^{b}$ \\ Bang-Ying Yu ${ }^{b}$,Bo-Cheng Lin $^{b}$, and Hsuen-Li Chen *a \\ ${ }^{a}$ Department of Materials Science and Engineering, National Taiwan University, \\ No. 1, Sec. 4, Roosevelt Road, Taipei 10617, Taiwan \\ ${ }^{\mathrm{b}}$ Materials and Electro-Optics Research Division, National Chung-Shan Institute of \\ Science and Technology, P.O. Box 90008-8-1, Lungtan, Taoyuan 32599, Taiwan \\ *Corresponding author \\ E-mail: hsuenlichen@ntu.edu.tw \\ Telephone: $+886-2-3366-3240$
}




\section{Contents}

1. Measurements of more positions on $\mathrm{SiC}$ wafers

2. The determination of the intensity of the two FTA doublet modes

3. Identification of the surface polarity of $\mathrm{SiC}$ by using a Raman system having a $473 \mathrm{~nm}$ excitation laser 


\section{Measurements of more positions on $\mathrm{SiC}$ wafers}

To clearly prove that we can identify the surface polarity of $\mathrm{SiC}$ by visible light based Raman spectroscopy, we also measured the Raman signals from many positions on different $4 \mathrm{H}-\mathrm{SiC}$ wafers. Figure $\mathrm{S} 1$ displays the photographs of the $4 \mathrm{H}-\mathrm{SiC}$ wafers, named sample A to sample C. The grid points, numbers, and letters represent the measured positions. Figure S2 presents the Raman peak intensity ratios of the signals at 210 and $203 \mathrm{~cm}^{-1}$ obtained from the $\mathrm{C}$ and $\mathrm{Si}$ faces at the each position of the three 4H-SiC wafers. Obviously, we found that the peak intensity ratio of the signals at 210 and $203 \mathrm{~cm}^{-1}$ was always higher when measured on Si face than on the $\mathrm{C}$ face, for all positions on the three different $\mathrm{SiC}$ wafers. Therefore, the result provides clear and convincing evidence that we can identify the surface polarity of SiC by using visible laser-based Raman spectroscopy. 

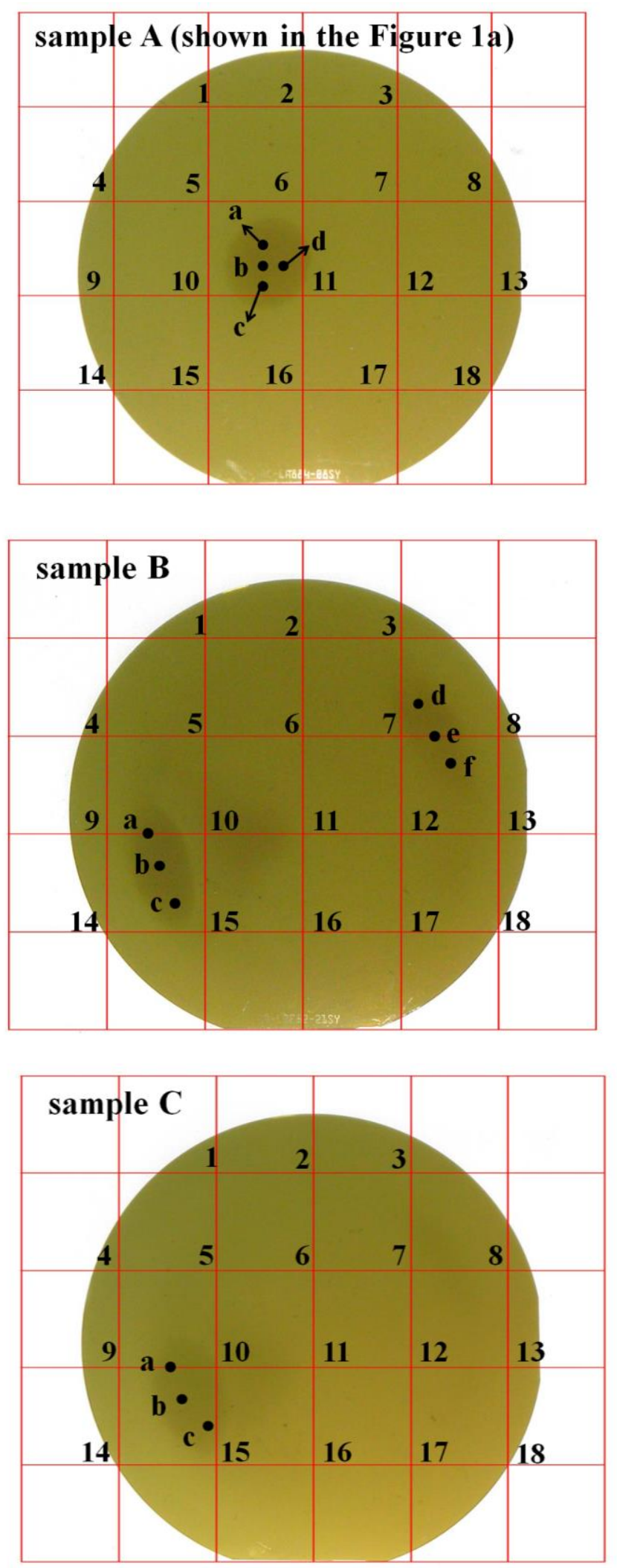

Figure S1. Photographs of the $4 \mathrm{H}-\mathrm{SiC}$ wafers measured in this study. 

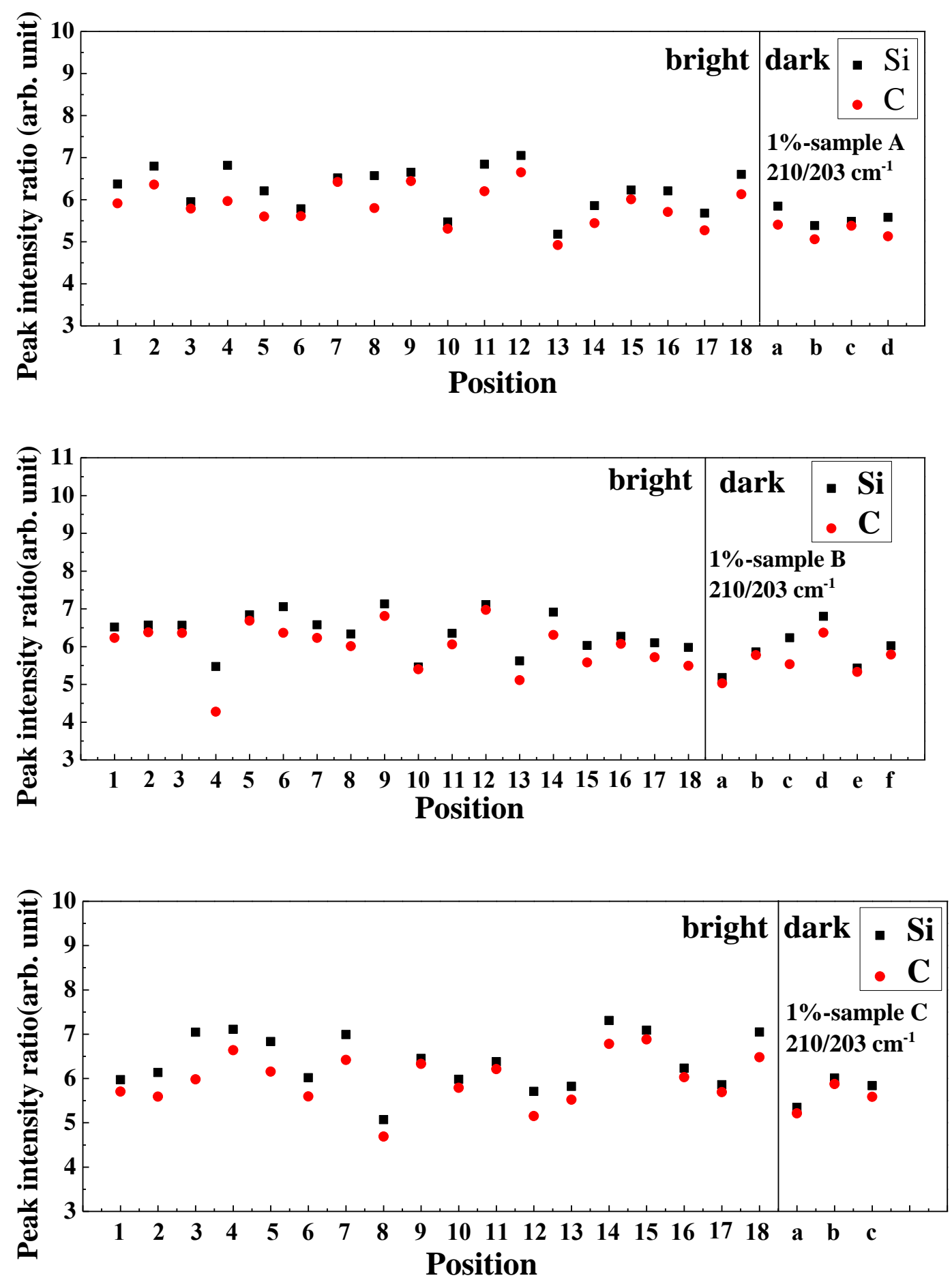

Figure S2. The Raman intensity ratios of the signals at 210 and $203 \mathrm{~cm}^{-1}$ on the $\mathrm{Si}$ 


\section{The determination of the intensity of the two FTA doublet modes}

As shown in Figure 5a and 5c, the Raman spectra of two FTA doublet modes do not display flat backgrounds. Therefore we took a simple baseline removal method (Figure S3) to determine the intensity of the two FTA doublet modes. Similar approach to determine the intensities of peaks was used in previous literature. ${ }^{\mathrm{S} 1}$

1. Take the Raman spectra in Figure 5c as an example, we first linearly fitted the Raman spectra of the background signals at both sides of the two FTA doublet modes, as the blue lines shown in Figure S3.

2. Then, we connected two close endpoints of blue lines to estimate a baseline, as the green line shown in Figure S3.

3. After the above operation, we can calculate the Raman intensities of the peaks at 203 and $210 \mathrm{~cm}^{-1}$ by subtracting an estimated baseline, as shown in Figure S3.

REFERENCES

(S1) Jiang, J. L.; Wang, Q.; Huang, H.; Zhang, X.; Wang, Y. B.; Geng, Q. F. Microstructure Evolution Induced by Ultraviolet Light Irradiation in Ti-Si Codoped Diamond-like Carbon films. J. Inorg. Mater. 2014, 29, 941-946. 


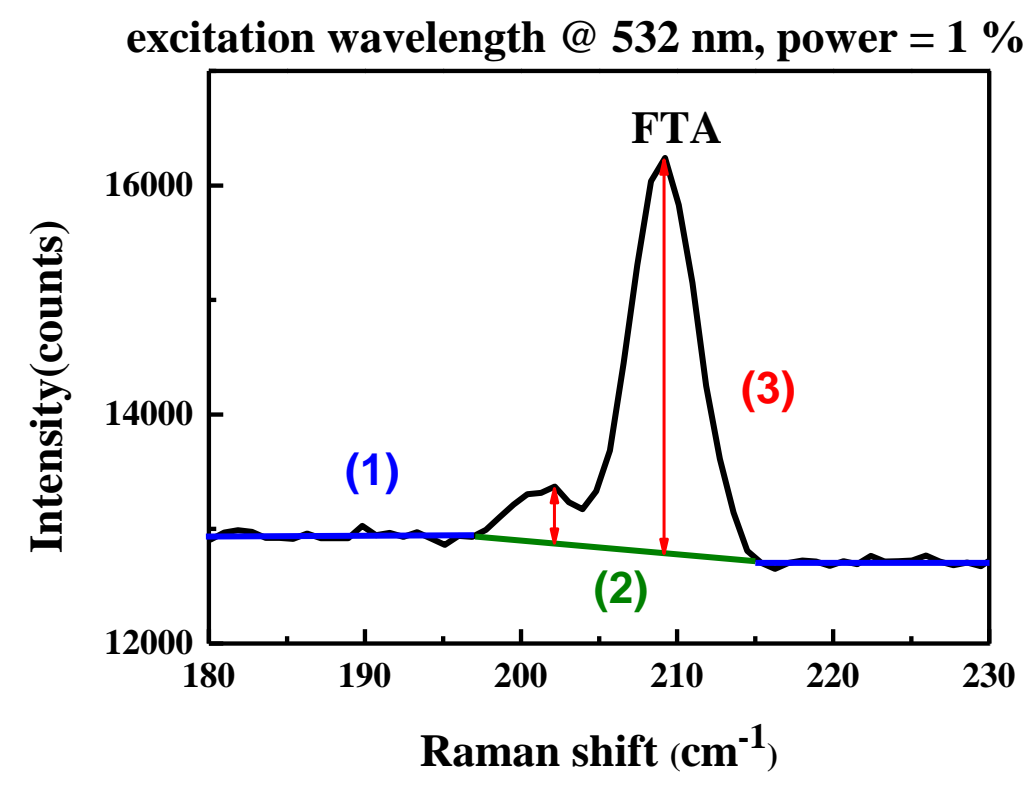

Figure S3. The baseline removal method we adopted in this study to determine the intensities of the two FTA doublet modes. 
Identification of the surface polarity of $\mathrm{SiC}$ by using a Raman system having a

\section{$473 \mathrm{~nm}$ excitation laser}

To confirm the idea of using visible laser to identify the surface polarity of SiC, we further employed another micro Raman system using a $473 \mathrm{~nm}$ excitation laser to confirm the measured results. Micro-Raman spectra of the $\mathrm{SiC}$ wafer were measured using a commercial micro-Raman microscope (UniRAM, UniNanoTech) equipped with a monochromator having a focal length of $30 \mathrm{~cm}$. The wavelength of the excitation laser line was fixed at $473 \mathrm{~nm}$. The laser beam was focused by a $50 \times$ objective having a numerical aperture of 0.55 . The power of the excitation laser was fixed at $100 \mathrm{~mW}$. The spot size of the excitation laser was approximately $0.86 \mu \mathrm{m}^{2}$; the integration time was $75 \mathrm{~s}$.

Figure S4 displays a photograph of the micro-Raman system having a $473 \mathrm{~nm}$ excitation laser. Figure S5 displays a Raman spectrum of 4H-SiC (sample A, position 1, Si face) obtained from an excitation laser having a wavelength of $473 \mathrm{~nm}$ with an attenuated intensity of $1 \%$. The Raman spectrum also appeared two optical phonon peaks at 203 and $778 \mathrm{~cm}^{-1}$, corresponding to the FTA and FTO vibration modes of 4H-SiC, respectively. Figure S6 displays the FTA mode of a 4H-SiC wafer excited by a $473-\mathrm{nm}$ laser with an attenuated intensity of $1 \%$. Notably, the signal of the FTA mode still appeared of a doublet, with a sharp peak at $203 \mathrm{~cm}^{-1}$ and a weaker peak at 
$194 \mathrm{~cm}^{-1}$. To identify the surface polarity of $\mathrm{SiC}$, we measured the Raman spectra of 18 positions in bright region and 4 positions in dark region, as displayed in the photograph of the $4 \mathrm{H}-\mathrm{SiC}$ (sample A) in Figure $\mathrm{S} 1$. We observed and calculated the peak intensity ratios between the signals at 203 and $194 \mathrm{~cm}^{-1}$ at various positions, as displayed in Figure S7. We noted that the peak intensity ratios of the signals at 203 and $194 \mathrm{~cm}^{-1}$ were always higher when measured on Si face than on the $\mathrm{C}$ face, for all 22 positions (1-18 in bright region, a- $\mathrm{d}$ in dark region). This feature is the same as the experimental result that we demonstrated by using an excitation laser having a wavelength of $532 \mathrm{~nm}$.

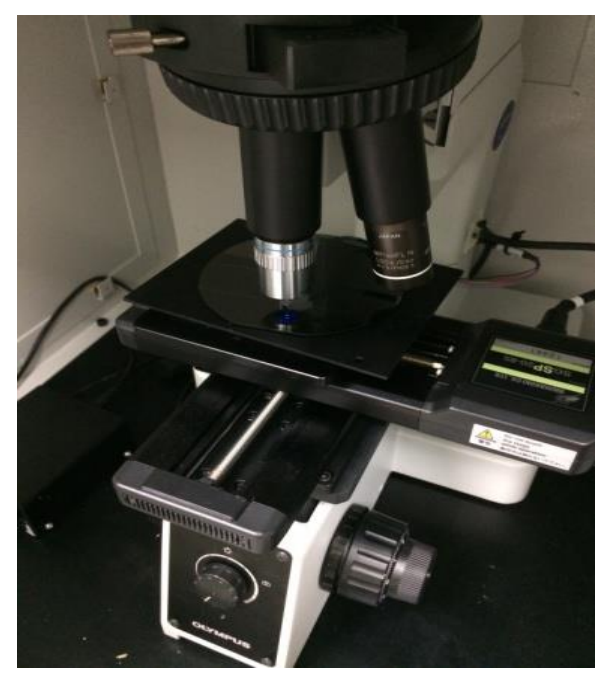

Figure S4. The photograph of the micro-Raman system having a $473 \mathrm{~nm}$ excitation laser used to characterize the SiC wafers. 


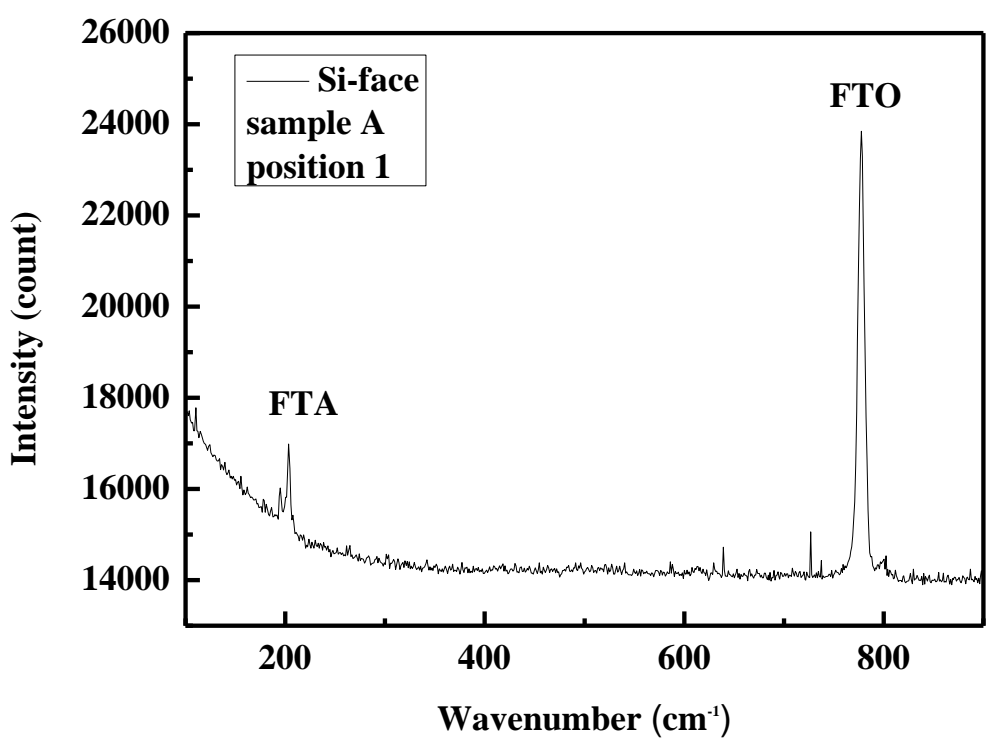

Figure S5. The Raman spectrum of 4H-SiC (sample A, position 1, Si face). 


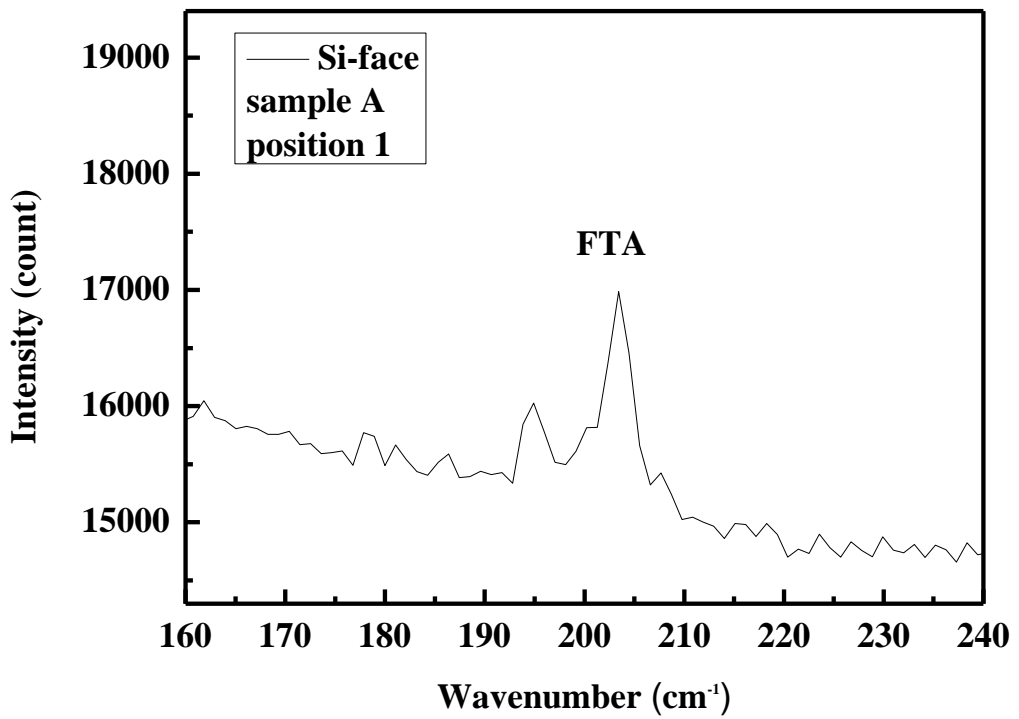

Figure S6. The FTA mode of a 4H-SiC wafer excited by a 473-nm laser. 


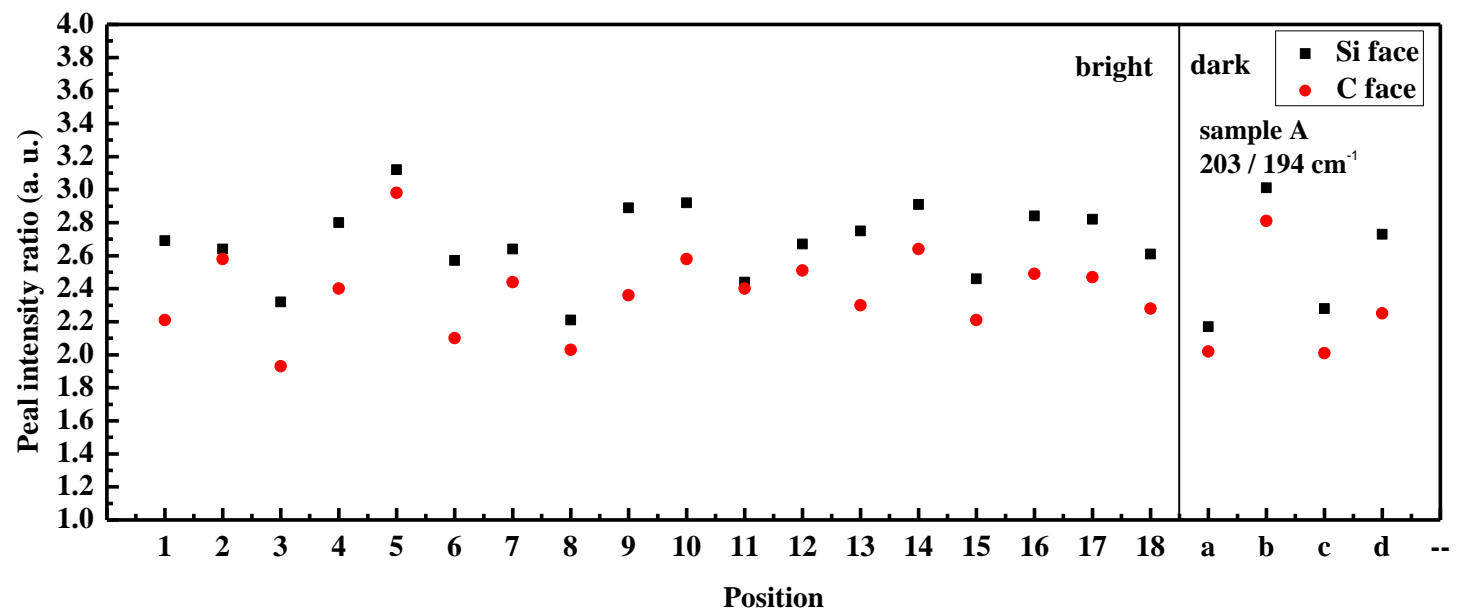

Figure S7. The Raman peak intensity ratios of the signals at 203 and $194 \mathrm{~cm}^{-1}$ obtained from the $\mathrm{C}$ and $\mathrm{Si}$ faces at the each position of the $4 \mathrm{H}-\mathrm{SiC}$ wafer (sample A). 